\title{
A Unified Linear Camera Calibration Method Using Two Parallel Calibration Planes
}

\author{
Koichiro DEGUCHI* and Iwao MoRISHITA*
}

\begin{abstract}
In this paper, we examine the two plane camera calibration model in terms of perspective mapping, and present a new effective camera calibration method by taking the advantage of the conventional method and modifying it based on the exact perspective imaging. It also takes the advantage that each of intrinsic and extrinsic camera parameters can be obtained by solving linear equations only. So that the solutions will be provided uniquely and stably under some conditions, and which can be definitely specified.

In our method a priori knowledge on camera intrinsic parameters is only physical pixel sizes. Exact focal length of the lens, the position of image center or optical center, and the camera pose are unknown and to be calibrated. This is a practical assumption for common commercial TV camera systems.

For the cases where non-linear lens distortions must be taken into account, our method can be extended and their parameters are calibrated also using only linear solutions.

It is shown that higher performance and accurate calibration can be achieved by this method theoretically and experimentally.
\end{abstract}

Key Words : computer vision, camera calibration, pose estimation, two-plane method

\section{Introduction}

Martins et $a l .{ }^{1)}$ proposed an effective camera calibration method named "Two plane method", and Gremban et $a l^{2)}$ improved it.

The authors of both papers claimed that their methods were effective under some nonlinear lens distortions because they did not employ the exact pin-hole camera model. However, it has been pointed out for this method that "since the formulas for the transformation between image and object coordinates is empirically based, it is not clear what kind of approximation is assumed"3).

Actually, as is shown later, their model which their techniques based on does not exactly hold physically. Moreover, their method is based on interpolation concept, so that it makes the error analysis difficult, and reliability of the methods is uncertain.

On the other hand, their method consists of only linear procedures. It is a great practical advantage that each intrinsic and extrinsic camera parameters can

\footnotetext{
* Faculty of Engineering, University of Tokyo, 7-3-1, Hongo, Bunkyo-ku, Tokyo, Japan

(Received June 24, 1992)

(Revised March 5, 1993)
}

be obtained by solving linear equations only, because the solutions are provided uniquely and stably, if conditions are definitely specified ${ }^{4), 5}$, and then the linear algorithm has a possibility of a definite analysis of error propagations in computations.

In this paper, we examine the two plane calibration model in terms of perspective mapping, and present a new effective method by taking the advantage of the two plane method and modifying it based on the exact perspective imaging.

In the new method, our a priori knowledge on camera intrinsic parameters is only physical pixel sizes. They are given by design specification data of CCD LSI and image digitizer. Exact focal length of the lens and the position of image center or optical center are unknown and to be calibrated. This is a practial assumption for common commercial TV camera.

In this method, two parallel calibration planes are prepared and a set of calibration points is collected in each plane (Fig. 1). At least four points on one of the planes, whose positions on the plane are exactly known, and at least two points on the other plane are needed, respectively.

This setup of calibration points may be realized by firstly setting the calibration plane at the first position 
and taking its first image, then, shifting it to the second position keeping it parallel to the plane at the first position, and taking its second image. Or, it may be effective to prepare a block-like object which has two parallel plane surfaces each of which contains at least four and two known points. It should be also noted that the positions of these calibration points in $3 \mathrm{D}$ space are given with sufficient accuracies practically required for individual systems. The extrinsic parameters of the camera are determined with respect to the coordinates based on these objects.

The total minimum number of calibration points required for our new algorithm is six, which is the same as the conventional method. It should be noted that any additional conditions are not needed for the improvement of the algorithm.

In the followings, in the Section 2, we review briefly the conventional two plane method, and point out the reason why the conventional method cannot achieve higher accuracies. Then, in the Section 3 and after, we present the new model, its geometric implication, and total camera calibration procedures. Finally, experimental results will be shown.

\section{Conventional Two-Plane Models}

Firstly, we summerize the conventional two plane method provided in 1) and 2). Their method did not assume a unique lens center. Their basic assumption is that, given an image point, two points in the 3D space, each of which is in each of two calibration planes, respectively, and both of which make their
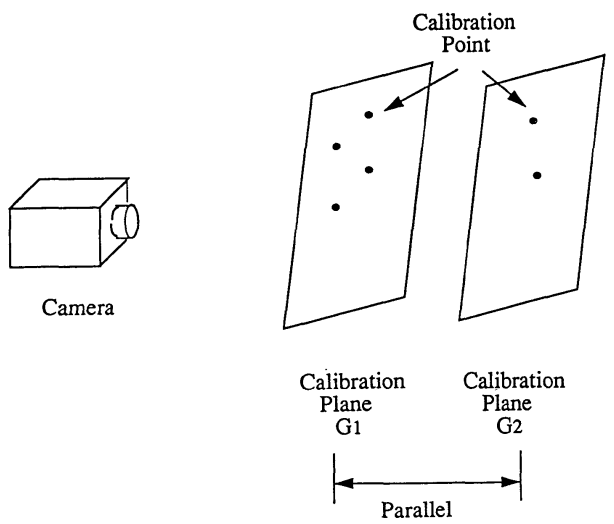

Fig. 1 Set-up of the calibration planes for the camera in the two-plane calibration method image at the given point in the image plane, are calculated. These two points define a line of sight of the given image point in the $3 \mathrm{D}$ space as shown in Fig. 2.

In their method, two parallel calibration planes are prepared and a set of calibration points is collected in each plane. Then, two so-called backprojection matrices which transform the position of a given point in the image plane into two positions of points in respective two calibration planes are determined. Using these two back-projection matrices, a line of sight of the given image point is defined as the line in the $3 \mathrm{D}$ space passing through these two points.

Many types of interpolation techniques have been proposed to define and determine the back-projection matrices for the given image point from the set of calibration points in the planes. Here, we summarize the simplest one called the linear or the linear spline interpolation.

Firstly, $x, y$ and $z$ axes of the base frame in 3D space are employed as both of the calibration planes are parallel to the $x-y$ plane. The position of the first calibration plane is given as $z=d_{1}$, and the second $z$ $=d_{2}$. The position of the image plane may be left unknown.

For the determination of a line of sight of a given image point $\boldsymbol{q}$, which has a homogeneous representation of $(r, c, 1)^{t}$ of image coordinates, $n$ calibration points are selected in the first calibration plane $G_{1}$ whose images are located around $\boldsymbol{q}$ in the image plane. And also for the second calibration plane $G_{2}, n$

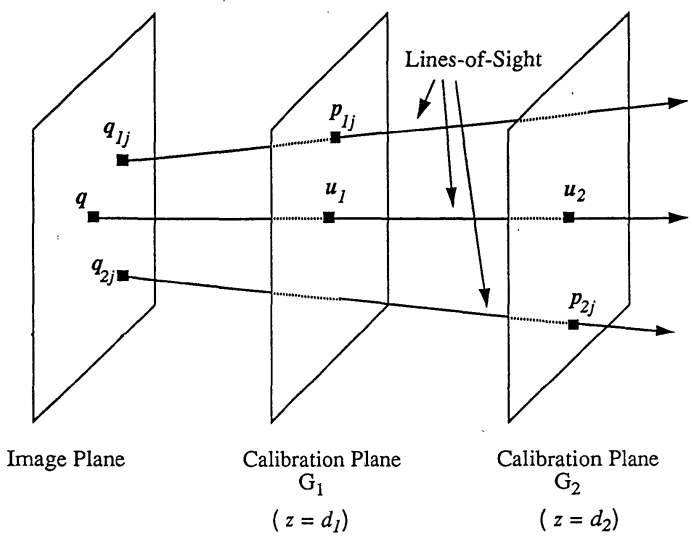

Fig. 2 Camera model in the two-plane method 
calibration points are selected similarly.

Let the $3 \mathrm{D}$ coordinates of the calibration points be denoted as

$$
\boldsymbol{p}_{i j}=\left(x_{i j}, y_{i j}, d_{i}\right)^{t}
$$

for $i=1$ and 2 according that the point belongs to the planes $G_{1}$ or $G_{2}$, and where $j$ is the index of points from 1 to $n$. And let the position of the image of the point $\boldsymbol{q}_{i j}$ be denoted as

$$
\boldsymbol{q}_{i j}=\left(r_{i j}, c_{i j}, 1\right)^{t}
$$

In the linear and linear spline method, the transformations between $\boldsymbol{p}_{i j}$ and $\boldsymbol{q}_{i j}$ are assumed to be expressed linearly as

$$
\boldsymbol{p}_{i j}=A_{i} \boldsymbol{q}_{i j} \quad(j=1,2, \cdots, n)
$$

where $A_{i}(i=1,2)$ are $3 \times 3$ matrices.

For the set of $n$ collected points, the relation can be expressed as

$$
P_{i}=A_{i} Q_{i} \quad(i=1,2)
$$

where $P_{i}=\left[\boldsymbol{p}_{i 1}, \boldsymbol{p}_{i 2}, \cdots, \boldsymbol{p}_{i n}\right]$ and $Q_{i}=\left[\boldsymbol{q}_{i 1}, \boldsymbol{q}_{i 2}, \cdots, \boldsymbol{q}_{i n}\right]$.

At least, three points per plane are needed, respectively, to determine the $A_{1}$ and $A_{2}(i . e . n \geq 3)$. After $A_{i}$ 's are determined, an image point $\boldsymbol{q}$ in the image plane can be "back-projected" into the planes $G_{1}$ and $G_{2}$, respectively, as

$$
\boldsymbol{u}_{i}=A_{i} \boldsymbol{q} \quad(i=1,2)
$$

Then, using the $\boldsymbol{u}_{1}$ and $\boldsymbol{u}_{2}$, the line of sight corresponding to the image point $\boldsymbol{q}$ is determined as the line passing through both of them.

To back-project the given point $\boldsymbol{q}$, some selection methods of $\boldsymbol{q}_{i j}$ 's for the determination of $A_{i}$ 's were proposed. One of the methods the linear spline interpolation method, is to use three nearest $\boldsymbol{q}_{i j}$ 's to the $\boldsymbol{q}$ (when $n=3$ ) as shown in Fig. 3. Then, $A_{i}$ is given as

$$
A_{i}=P_{i} Q_{i}^{-1}
$$

When more points are collected and a least square method is employed, the solution will be,

$$
A_{i}=P_{i} Q_{i}^{-}=P_{i} Q_{i}^{t}\left(Q_{i} Q_{i}^{t}\right)^{-1}
$$

and this is called the linear interpolation method.

When lines of sight for several image points have been obtained, the optical center of the imaging system is determined as the point where the bundle of lines of sight encounter. The details of these procedures are skipped here and will be described later, because these stages are effective for our new algorithm.

There is a total of 18 coefficients in the matrices $A_{i}$ (nine for each $i$ ) to be determined. In the case where

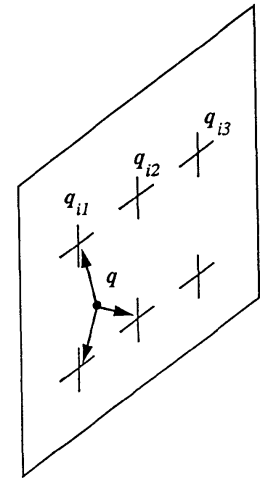

Image Plane

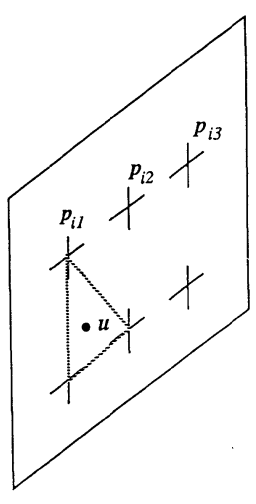

Calibration Plane $G_{i}$
Fig. 3 Interpolation in the Linear Spline Method

the planes are parallel to the $x-y$ plane so that the third row of the two matrices is known to be $\left(0,0, d_{i}\right)$, the number of coefficients is reduced to 12 ( $\mathrm{six}$ for each $i$ ). That is, the matrix $A_{i}$ has the form of

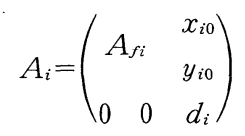

This means that in this scheme the mapping between $2 \mathrm{D}$ coordinates of the image point $(r, c)^{t}$ and the $2 \mathrm{D}$ coordinates of calibration point $\left(x_{i}, y_{i}\right)$ in the calibration plane $G_{i}$ is assumed to be given as

$$
\left(\begin{array}{l}
x_{i} \\
y_{i}
\end{array}\right)=A_{f i}\left(\begin{array}{l}
r \\
c
\end{array}\right)+\left(\begin{array}{l}
x_{i 0} \\
y_{i 0}
\end{array}\right)
$$

This mapping is of Affine transformation. Therefore, in the conventional two plane methods, the imaging of a pattern on a plane in $3 \mathrm{D}$ space is assumed to be an Affine transformation.

However, it is known that the perspective transformation between $2 \mathrm{D}$ planes in $3 \mathrm{D}$ space does not agree with Affine transformation. Only if the object plane and the image plane are nearly parallel, or if the set of points span within a local small spatial region, the perspective transformation is well approximated by the Affine transformation. For the former case, the approximation error depends on the slant of the image plane to the global $z$-axis. For the latter case, it depends on the arrangement and the density of the calibration points given on the calibration planes. These are the reasons why the performance and accuracy of the conventional two plane method becomes uncertain.

It is also known ${ }^{6) 7}$ that a perspective transforma- 
tion between two given planes in $3 \mathrm{D}$ space will be defined uniquely when at least four pairs of point correspondences are given. The model on which the conventional method based, where the relational matrix $A_{i}$ are determined with three points for each, lacks in the degree of freedom to express the mapping sufficiently. Therefore, it is clear that a new formulation of the two plane model is needed.

\section{A Unified Model of Two Plane Camera Calibration}

To modify the two plane method and present a new algorithm, here we formulate a unified camera model.

We define three coordinate systems ; world coordinates, camera coordinates, and image coordinates, as shown in Fig. 4.

The world coordinate system $O_{o}-X Y Z$ is based on calibration planes. The calibration planes are placed so as to be $Z=Z_{i}$, and calibration points are given with $(X, Y)$ coordinates.

The camera coordinate system is represented by $O_{i}-x y z . O_{i}$ is the optical center and the $z$-axis is the optical axis of the camera. The image plane is corresponding to $z=F$, where $F$ is the focal length. On the image plane, image coordinates are given as $(u, v)$ independently with $(x, y, \dot{z})$ coordinates, but the directions of $x$ and $y$-axes are coincident with $u$ and $v$-axes, respectively. The optical axis, $z$-axis, intersects the image plane at $\left(u_{0}, v_{0}\right)$, which corresponds to the image center on the image plane.

Of course, the position of $O_{i}$ and the direction of $z$-axis with respect to $O_{o}-X Y Z$ system, as well as $F$ and $\left(u_{0}, v_{0}\right)$, are unknown to be calibrated.

The two $O_{o}-X Y Z$ and $O_{i}-x y z$ systems can be related by a rotation matrix $R$ and a translation vector $\boldsymbol{t}$ as

$$
\left(\begin{array}{l}
x \\
y \\
z
\end{array}\right)=R\left(\begin{array}{l}
X \\
Y \\
Z
\end{array}\right)+\boldsymbol{t}
$$

Then the perspective image of the point $(x, y, z)$ in camera coordinates is given as

$$
\begin{aligned}
& u=\left(1 / \rho_{u}\right) F \frac{x}{z}+u_{0} \\
& v=\left(1 / \rho_{v}\right) F \frac{y}{z}+v_{0}
\end{aligned}
$$

where $\rho_{u}$ and $\rho_{v}$ are scale factors based on the physical pixel sizes of the image plane.

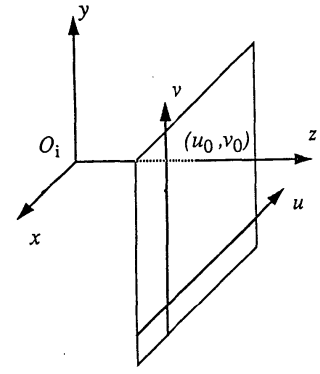

Image plane

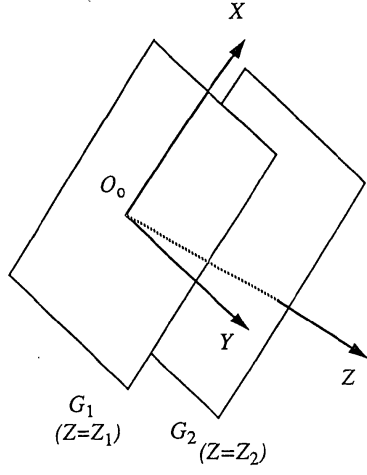

Calibration planes
Fig. 4 Coordinate systems of camera, object and image plane

Substituting (10) in (11) and rearranging and introducing the new variables, the transformation from the object coordinates $(X, Y, Z)$ to the image coordinates $(u, v)$ can finally be expressed as

$$
\begin{aligned}
& u=\frac{a_{1} X+a_{2} Y+a_{3} Z+T_{1}}{c_{1} X+c_{2} Y+c_{3} Z+T_{3}} \\
& v=\frac{b_{1} X+b_{2} Y+b_{3} Z+T_{2}}{c_{1} X+c_{2} Y+c_{3} Z+T_{3}}
\end{aligned}
$$

For all the calibration points in $G_{1}, Z=Z_{1}$, and for the points in $G_{2}, Z=Z_{2}$, which are constants and given. Therefore, denoting the 2D coordinates of the points in $G_{1}$ as $\left(X_{1}, Y_{1}\right)$ and their image coordinates as $\left(u_{1}\right.$, $\left.v_{1}\right)$, and the $2 \mathrm{D}$ coordinates in $G_{2}$ an $\left(X_{2}, Y_{2}\right)$ and their image coordinates as $\left(u_{2}, v_{2}\right)$, we have

$$
\begin{aligned}
& u_{1}=\frac{a_{1} X_{1}+a_{2} Y_{1}+a_{31}}{c_{1} X_{1}+c_{2} Y_{1}+c_{31}}, \\
& v_{1}=\frac{b_{1} X_{1}+b_{2} Y_{1}+b_{31}}{c_{1} X_{1}+c_{2} Y_{1}+c_{31}} \\
& u_{2}=\frac{a_{1} X_{2}+a_{2} Y_{2}+a_{32}}{c_{1} X_{2}+c_{2} Y_{2}+c_{32}}, \\
& v_{2}=\frac{b_{1} X_{2}+b_{2} Y_{2}+b_{32}}{c_{1} X_{2}+c_{2} Y_{2}+c_{32}}
\end{aligned}
$$

Because $c_{31}$ in (13) is never equal to zero, setting $c_{31}$ $=1$, we have equations for the variable $\left\{a_{1}, a_{2}, a_{31}, b_{1}\right.$, $\left.b_{2}, b_{31}, c_{1}, c_{2}\right\}$,

$$
\begin{aligned}
& \left(\begin{array}{cccccccc}
X_{1} & Y_{1} & 1 & 0 & 0 & 0 & -u_{1} X_{1} & -u_{1} Y_{1} \\
0 & 0 & 0 & X_{1} & Y_{1} & 1 & -v_{1} X_{1} & -v_{1} Y_{1}
\end{array}\right)\left(\begin{array}{c}
a_{1} \\
a_{2} \\
a_{31} \\
\vdots \\
c_{2}
\end{array}\right) \\
& =\left(\begin{array}{l}
u_{1} \\
v_{1}
\end{array}\right)
\end{aligned}
$$


If we have at least four points in $G_{1}$, and if any three of which are not co-linear, we can solve (15) and obtain the coefficients $\left\{a_{1}, a_{2}, a_{31}, b_{1}, b_{2}, b_{31}, c_{1}, c_{2}\right\}$ to transform $G_{1}$ to the image plane.

Then, the remaining unknown parameters $\left\{a_{32}, b_{32}\right.$, $\left.c_{32}\right\}$ for $G_{2}$ are obtained by substituting above obtained coefficients into (14) and reformulating it as

$$
\begin{aligned}
-a_{32}+u_{2} c_{32} & =a_{1} X_{2}+a_{2} Y_{2}-u_{2}\left(c_{1} X_{2}+c_{2} Y_{2}\right) \\
-b_{32}+v_{2} c_{32} & =b_{1} X_{2}+b_{2} Y_{2}-v_{2}\left(c_{1} X_{2}+c_{2} Y_{2}\right)
\end{aligned}
$$

At least two calibration points in $G_{2}$ and their image points are needed to obtain $\left\{a_{32}, b_{32}, c_{32}\right\}$.

Therefore, at least six points, four of which are in one plane and two in the other plane, are required to determine the transformation from the object planes to the image. If more points are available, the least square solution can be employed to reduce the errors.

After the coefficients of transformation of "projection" into the image plane are determined, we can "back-project" a given image point $(u, v)$ into the planes $G_{1}$ or $G_{2}$ by using the next relation derived from (13) and (14);

$$
\begin{aligned}
& X_{i}=\frac{A_{1 i} u+A_{2 i} v+A_{3 i}}{C_{1} u+C_{2} v+C_{3}}, \\
& Y_{i}=\frac{B_{1 i} u+B_{2 i} v+B_{3 i}}{C_{1} u+C_{2} v+C_{3}}
\end{aligned}
$$

where $i=1,2$ and

$$
\begin{aligned}
& A_{1 i}=c_{3 i} b_{2}-b_{3 i} c_{2}, A_{2 i}=a_{3 i} c_{2}-c_{3 i} a_{2} \\
& A_{3 i}=b_{3 i} a_{2}-a_{3 i} b_{2}, \quad B_{1 i}=-c_{3 i} b_{1}+b_{3 i} c_{1}, \\
& B_{2 i}=-a_{3 i} c_{1}+c_{3 i} a_{1}, B_{3 i}=-b_{3 i} a_{1}+a_{3 i} b_{1} \\
& C_{1}=b_{1} c_{2}-b_{2} c_{1}, \quad C_{2}=c_{1} a_{2}-c_{2} a_{1}, \quad C_{3}=a_{1} b_{2}-a_{2} b_{1}
\end{aligned}
$$

The $3 \mathrm{D}$ coordinates $\left(X_{1}, Y_{1}, Z_{1}\right)$ and $\left(X_{2}, Y_{2}, Z_{2}\right)$ given by (17) are the corresponding back-projected points on $G_{1}$ and $G_{2}$, respectively.

\section{Camera Calibration by Two Plane Back Projection}

\section{1 Optical Center}

For a given image point, we just provided the corresponding back-projected points in $G_{1}$ and $G_{2}$. Let those points be denoted as $\boldsymbol{u}_{1}\left(X_{1}, Y_{1}, Z_{1}\right)$ and $\boldsymbol{u}_{2}\left(X_{2}\right.$, $\left.Y_{2}, Z_{2}\right)$, respectively. The line passing those points $\boldsymbol{u}_{1}$ and $\boldsymbol{u}_{2}$ in $3 \mathrm{D}$ space means the line of sight of the image point $(u, v)$.

The optical center $O_{i}$ of the imaging system is defined to be the point where all the lines of sight pass through. The coordinates of $O_{i}$ with respect to the object coordinate system $O_{0}-X Y Z$ can be computed through the two step computation. Determining the lines of sight firstly and then the point they are passing, the optical center is obtained by solving a set of linear equations.

This can be done by following the technique proposed in 2). Here we summerize it.

Theoretically, the optical center is determined by two lines of sight as their crossing point. But this computation is sensitive to the computational roundoff errors. So, we define the "effective" optical center as the nearest point from each line of sight (Fig.5).

Let the distance in 3D space between a point $(x, y$, $z)^{t}$ and a line which passes $\left(x_{1}, y_{1}, z_{1}\right)^{t}$ with a direction vector $(a, b, c)^{t}$ (where $a^{2}+b^{2}+c^{2}=1$ ) be denoted as $d$. Then $d$ is given as

$$
d^{2}=\left|\begin{array}{cc}
y-y_{1} & z-z_{1} \\
b & c
\end{array}\right|^{2}+\left|\begin{array}{cc}
z-z_{1} & x-x_{1} \\
c & a
\end{array}\right|^{2}+\left|\begin{array}{cc}
x-x_{1} & y-y_{1} \\
a & b
\end{array}\right|^{2}
$$

Now, let the calibration points in $G_{1}$ and $G_{2}$ be used as $\left(x_{i}, y_{i}, z_{i}\right)$ 's, and the directional vectors of their lines of sight be $\left(a_{i}, b_{i}, c_{i}\right)$ 's. Also, let the coordinates of the effective optical center be $\left(f_{x}, f_{y}, f_{z}\right)$, and the sum of square of the distances from it to all the lines of sight be $D_{c}\left(D_{c}=\sum d_{i}^{2}\right)$. Then, $\left(f_{x}, f_{y}, f_{z}\right)$ can be obtained by minimizing $D_{c}$, so that,

$$
\partial D_{c} /\left.\partial x\right|_{x=f x}=\partial D_{c} /\left.\partial y\right|_{y=f y}=\partial D_{c} /\left.\partial z\right|_{z=f_{z}}=0
$$

Rewriting these equations in matrix-vector forms, we have

$$
\boldsymbol{h}=H \boldsymbol{f}
$$

where

$$
\begin{aligned}
& \boldsymbol{h}=\left(\sum\left(c k_{2}-b k_{3}\right), \quad \sum\left(a k_{3}-c k_{1}\right), \quad \sum\left(b k_{1}-a k_{2}\right)\right)^{t} \\
& \left(k_{1}=z_{1} b-y_{1} c, \quad k_{2}=x_{1} c-z_{1} a, k_{3}=y_{1} a-x_{1} b\right) \\
& H=\left(\begin{array}{ccc}
\Sigma\left(b^{2}+c^{2}\right) & -\sum a b & -\sum a c \\
-\sum a b & \sum\left(a^{2}+c^{2}\right) & -\sum b c \\
-\sum a c & -\sum b c & \sum\left(a^{2}+b^{2}\right)
\end{array}\right) \\
& \boldsymbol{f}=\left(f_{x}, f_{y}, f_{z}\right)^{t}
\end{aligned}
$$

(Subscripts $i$ are omitted for the simplicity.)

Therefore, the $\left(f_{x}, f_{y}, f_{z}\right)^{t}$ will be given by

$$
\boldsymbol{f}=H^{-1} \boldsymbol{h}
$$

\section{2 Optical Axis and Pose of Image Plane}

The optical axis and the pose of the image plane are given as to fit the image points to the points at which the corresponding lines of sight across the image plane (Fig. 6). However, the actual available 


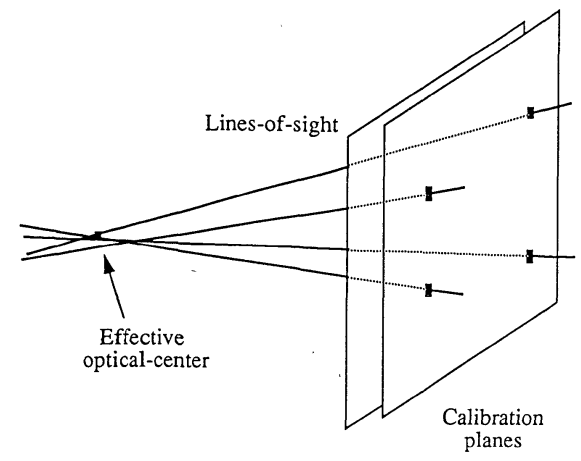

Fig. 5 Determination of the effective optical center

algorithm to determine it has not been presented yet in 1$) \sim 5$ ). Here, we present a new scheme for it.

In this subsection, all 3D coordinate vectors are defined based on the object coordinate system $O_{o}-X Y Z$. Firstly, we define and list the additional vectors and parameters used here.

- $\boldsymbol{p}=\left(p_{x}, p_{y}, p_{z}\right)^{t}:$ a point in 3D space,

- $l$ : unit directional vector from optical center to the point $\boldsymbol{p}$,

- $\boldsymbol{P}$ : crossing point of the line-of-sight of $\boldsymbol{l}$ onto the image plane,

- $\boldsymbol{P}^{\prime}$ : spatial position of image point $(u, v)$ of $\boldsymbol{p}$,

- $\boldsymbol{g}$ : unit directional vector of optical axis,

- $\boldsymbol{r}=\left(r_{x}, r_{y}, r_{z}\right)^{t}, \boldsymbol{c}=\left(c_{x}, c_{y}, c_{z}\right)^{t}$ : vectors defining spatial sizes and directions of of one pixel in the image plane in $u$ and $v$ directions, respectively.

These are shown in Fig. 7.

The unit directional vector of the line of sight for a point $\boldsymbol{p}$ is given as

$$
\boldsymbol{l}=\frac{\boldsymbol{p}-\boldsymbol{f}}{|\boldsymbol{p}-\boldsymbol{f}|}
$$

Now, from $(\boldsymbol{P}-\boldsymbol{f}) \cdot \boldsymbol{g}=F$, which is the focal length,

$$
\boldsymbol{P}=\boldsymbol{f}+\frac{F}{(\boldsymbol{l} \cdot \boldsymbol{g})} \cdot \boldsymbol{l}
$$

On the other hand, $\boldsymbol{P}^{\prime}$ is given as

$$
\boldsymbol{P}^{\prime}=\boldsymbol{f}+F \boldsymbol{g}+\left(u-u_{0}\right) \boldsymbol{r}+\left(v-v_{0}\right) \boldsymbol{c}
$$

$\boldsymbol{P}$ does not necessarily coincident with $\boldsymbol{P}^{\prime}$. Then, we denote the difference between $\boldsymbol{P}$ and $\boldsymbol{P}^{\prime}$ on the image plane with $\Delta$, that is,

$$
\Delta=\left|\boldsymbol{P}-\boldsymbol{P}^{\prime}\right|
$$

Then, $\Delta^{2}$ is given as

$$
\Delta^{2}=F^{2}\left(\frac{1}{(\boldsymbol{l} \cdot \boldsymbol{g})^{2}}-1\right)-2 F\left(u-u_{0}\right) \frac{(\boldsymbol{l} \cdot \boldsymbol{r})}{(\boldsymbol{l} \cdot \boldsymbol{g})}
$$

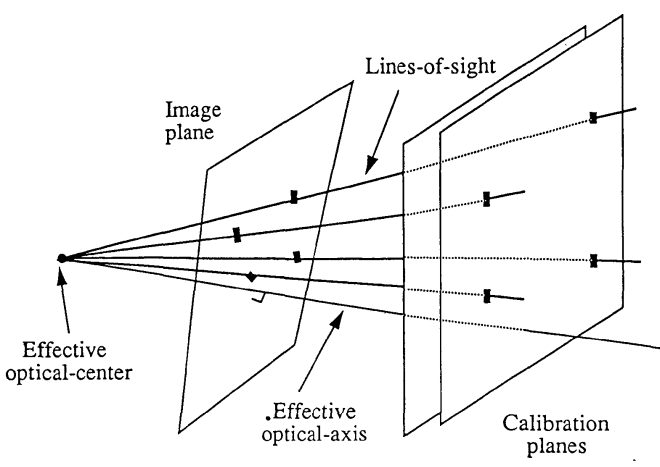

Fig. 6 Determination of the effective optical axis

$$
\begin{aligned}
& -2 F\left(v-v_{0}\right) \frac{(\boldsymbol{l} \cdot \boldsymbol{c})}{(\boldsymbol{l} \cdot \boldsymbol{g})}+\left(u-u_{0}\right)^{2}|\boldsymbol{r}|^{2} \\
& +\left(v-v_{0}\right)^{2}|\boldsymbol{c}|^{2} \\
= & \left(\left(u-u_{0}\right)|\boldsymbol{r}|-F \frac{\left(\boldsymbol{l} \cdot \boldsymbol{r}^{\prime}\right)}{(\boldsymbol{l} \cdot \boldsymbol{g})}\right)^{2} \\
& +\left(\left(v-v_{0}\right)|\boldsymbol{c}|-F \frac{\left(\boldsymbol{l} \cdot \boldsymbol{c}^{\prime}\right)}{(\boldsymbol{l} \cdot \boldsymbol{g})}\right)^{2}
\end{aligned}
$$

(where $\boldsymbol{r}^{\prime}=\boldsymbol{r} /|\boldsymbol{r}|$, and $\boldsymbol{c}^{\prime}=\boldsymbol{c} /|\boldsymbol{c}|$ ).

Because $\boldsymbol{g}, \boldsymbol{r}^{\prime}$ and $\boldsymbol{c}^{\prime}$ make up an orthonormal coordinate system and $|\boldsymbol{l}|=1$,

$$
(\boldsymbol{l} \cdot \boldsymbol{g})^{2}+\left(\boldsymbol{l} \cdot \boldsymbol{r}^{\prime}\right)^{2}+\left(\boldsymbol{l} \cdot \boldsymbol{c}^{\prime}\right)=1
$$

For all the test point $\boldsymbol{p}_{i}(i=1, \cdots, N)$, let the total sum of their squared differences be $D_{p}$, that is, letting $\Delta_{i}=\left|\boldsymbol{P}_{i}-\boldsymbol{P}_{i}^{\prime}\right|$,

$$
\begin{aligned}
D_{p} & =\sum_{i} \Delta_{i}^{2} \\
& =\sum_{i}\left(\left(u_{i}-u_{0}\right)|\boldsymbol{r}|-F \frac{\left(\boldsymbol{l}_{i} \cdot \boldsymbol{r}^{\prime}\right)}{\left(\boldsymbol{l}_{i} \cdot \boldsymbol{g}\right)}\right)^{2}
\end{aligned}
$$

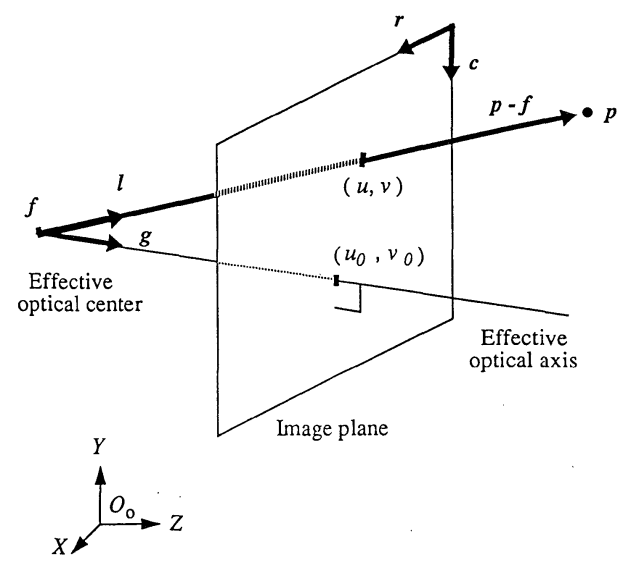

Fig. 7 Definitions and relations of spatial vectors used in the determination of effective optical axis and image coordinates 


$$
+\sum_{i}\left(\left(v_{i}-v_{0}\right)|\boldsymbol{c}|-F \frac{\left(\boldsymbol{l}_{i} \cdot \boldsymbol{c}^{\prime}\right)}{\left(l_{i} \cdot \boldsymbol{g}\right)}\right)^{2}
$$

Then, introducing new temporal unknown variables $\boldsymbol{R}=F \boldsymbol{r} /|\boldsymbol{r}|^{2}+u_{0} \boldsymbol{g}$ and $\boldsymbol{C}=F \boldsymbol{c} /|\boldsymbol{c}|^{2}+v_{0} \boldsymbol{g}$, becomes

$$
D_{p}=|\boldsymbol{r}|^{2} \sum_{i}\left(u_{i}-\frac{\left(\boldsymbol{l}_{i} \cdot \boldsymbol{R}\right)}{\left(\boldsymbol{l}_{i} \cdot \boldsymbol{g}\right)}\right)^{2}+|\boldsymbol{c}|^{2} \sum_{i}\left(v_{i}-\frac{\left(\boldsymbol{l}_{i} \cdot \boldsymbol{C}\right)}{\left(\boldsymbol{l}_{i} \cdot \boldsymbol{g}\right)}\right)^{2}
$$

If we employ the test points $\boldsymbol{p}_{i}$ so as their images are within region whose size is sufficiently small compared to the focal length $F$ (which is called "small image assumption" and this is a usual case), we may consider that $\left(\boldsymbol{l}_{i} \cdot \boldsymbol{g}\right)$ is constant for all the test point. For these case, the minimization of $D_{p}$ is established when $\Sigma_{i}\left(u_{i}\left(\boldsymbol{l}_{i} \cdot \boldsymbol{g}\right)-\left(\boldsymbol{l}_{i} \cdot \boldsymbol{R}\right)\right)^{2} \rightarrow \min$. and $\sum_{i}\left(v_{i}\left(\boldsymbol{l}_{i} \cdot \boldsymbol{g}\right)\right.$ $\left.-\left(\boldsymbol{l}_{i} \cdot \boldsymbol{C}\right)\right)^{2} \rightarrow \min$.

Now we need the ratios of the components of $\boldsymbol{g}$ to determine the direction of the optical axis. So, this can be achieved by setting $g_{z}=1$ and solving the next linear equation by the least square error method:

Denote the components of $\boldsymbol{R}$ and $\boldsymbol{C}$ with $\left(R_{x}, R_{y}, R_{z}\right)$ and $\left(C_{x}, C_{y}, C_{z}\right)$, respectively, and $\boldsymbol{l}_{i}$ with $\left(l_{i x}, l_{i y}, l_{I z}\right)$. Then, solve

$$
\begin{aligned}
& {\left[\begin{array}{cccccccc}
u_{1} l_{x 1} & u_{1} l_{y 1} & -l_{x 1} & -l_{y 1} & -l_{z 1} & 0 & 0 & 0 \\
v_{1} l_{x 1} & v_{1} l_{y 1} & 0 & 0 & 0 & -l_{x 1} & -l_{y 1} & -l_{z 1} \\
u_{2} l_{x 2} & u_{2} l_{y 2} & -l_{x 2} & -l_{y 2} & -l_{z 2} & 0 & 0 & 0 \\
v_{2} l_{x 2} & v_{2} l_{y 2} & 0 & 0 & 0 & -l_{x 2} & -l_{y 2} & -l_{z 2} \\
\vdots & \vdots & \vdots & \vdots & \vdots & \vdots & \vdots & \vdots \\
u_{n} l_{x n} & u_{n} l_{y n} & -l_{x n} & -l_{y n} & -l_{z n} & 0 & 0 & 0 \\
v_{n} l_{x n} & v_{n} l_{y n} & 0 & 0 & 0 & -l_{x n} & -l_{y n} & -l_{z n}
\end{array}\right]} \\
& \quad\left[\begin{array}{c}
g_{x} \\
g_{y} \\
R_{x} \\
R_{y} \\
R_{z} \\
C_{x} \\
C_{y} \\
C_{z}
\end{array}\right]=\left[\begin{array}{c}
u_{1} l_{z 1} \\
v_{1} l_{z 1} \\
u_{2} l_{z 2} \\
v_{2} l_{z 2} \\
\vdots \\
u_{n} l_{z n} \\
v_{n} l_{z n}
\end{array}\right]
\end{aligned}
$$

for given $n$ test points. The resulted solution $\boldsymbol{g}$ must be normalized as $|\boldsymbol{g}|=1$.

Using these $\boldsymbol{R}, \boldsymbol{C}$, and their definitions, and the fact that $(\boldsymbol{g} \cdot \boldsymbol{r})=(\boldsymbol{g} \cdot \boldsymbol{c})=0$, the image center coordinates are given as,

$$
\begin{aligned}
& u_{0}=(\boldsymbol{R} \cdot \boldsymbol{g}) \\
& v_{0}=(\boldsymbol{C} \cdot \boldsymbol{g})
\end{aligned}
$$

This $\left(u_{0}, v_{0}\right)$ is the crossing point of the optical axis on the image plane.

Then, using $\left(u_{0}, v_{0}\right)$, the focal length can be obtained as

$$
F=\sqrt{\left(u-u_{0}\right)^{2} /|\boldsymbol{r}|^{2}+\left(v-v_{0}\right)^{2} /|\boldsymbol{c}|^{2}} /(\boldsymbol{l} \cdot \boldsymbol{g})
$$

\section{Lens Distortion Models}

Our imaging model of (10), (11) and (12) are based on the exact pinhole model. The proposed technique is to determine the "effective" optical center and optical axis with the least mean square techniques. Therefore, it works well under some lens distortions as shown in the experiments later.

But, there may be cases where non-linear lens distortions must be taken into account. It has been believed that, when non-linear distortions were assumed, the determination of camera intrinsic parameters must involve non-linear iterative search ${ }^{3)}$, even in the two plane method by Martins et al. ${ }^{1)}$ In their method, to avoid this, two reference calibration planes must be parallel to image plane, which was not realistic as was pointed out.

However, our two plane camera calibration method can be carried out using only linear solutions while considering all lens distortions.

If there exist lens distortions, the left hand sides of the perspective imaging of (11) must be replaced as

$$
u \longrightarrow u+\delta u \text {, and } v \longrightarrow v+\delta v
$$

where $(\delta u, \delta v)$ are corrections to compensate for those distortions.

For well designed and manufactured lenses, it was reported and verified to be sufficient to take account the following components ${ }^{8,9)}$ :

$$
\begin{gathered}
\delta u=\underbrace{k_{1} u\left(u^{2}+v^{2}\right)}_{\text {radial }}+\underbrace{p_{1}\left(3 u^{2}+v^{2}\right)+2 p_{2} u v}_{\text {decentering }}+\underbrace{s_{1}\left(u^{2}+v^{2}\right)}_{\text {thin prism }} \\
\delta v=\underbrace{k_{1} v\left(u^{2}+v^{2}\right)}_{\text {radial }}+\underbrace{2 p_{1} u v+p_{2}\left(u^{2}+3 v^{2}\right)}_{\text {decentering }}+\underbrace{s_{2}\left(u^{2}+v^{2}\right)}_{\text {thin prism }}
\end{gathered}
$$

Substituting $(u, v)$ by $(u+\delta u, v+\delta v)$ into (17), we arrive at following general forms ;

$$
\begin{aligned}
& X_{1}=\sum_{i+j=0}^{3} A_{i j}^{(1)} u^{i} v^{j} / \sum_{i+j=0}^{3} C_{i j}^{(1)} u^{i} v^{j} \\
& Y_{1}=\sum_{i+j=0}^{3} B_{i j}^{(1)} u^{i} v^{j} / \sum_{i+j=0}^{3} C_{i j}^{(1)} u^{i} v^{j} \\
& X_{2}=\sum_{i+j=0}^{3} A_{i j}^{(2)} u^{i} v^{j} / \sum_{i+j=0}^{3} C_{i j}^{(2)} u^{i} v^{j} \\
& Y_{2}=\sum_{i+j=0}^{3} B_{i j}^{(2)} u^{i} v^{j} / \sum_{i+j=0}^{3} C_{i j}^{(2)} u^{i} v^{j}
\end{aligned}
$$


as the back-projection transformation.

Equations (40) and (41) constitute a set of linear equations on the coefficients $\left\{A_{i j}^{(k)}, B_{i j}^{(k)}, C_{i j}^{(k)}\right\}(k=1,2)$, and we can directly solve for them by linear computations. It is worth of noting that

$$
\begin{aligned}
& C_{i j}^{(1)}=0, \quad C_{i j}^{(2)}=0: \text { for } 0<i+j \leq 3 \\
& B_{i j}^{(1)}=0, \quad A_{i j}^{(2)}=0: \text { for } i+j=3
\end{aligned}
$$

With these transformation coefficients we can also map each image point onto the two calibration planes $G_{1}$ and $G_{2}$ to get two corresponding object points. Connecting those two points will result in the line of sight for the given image point. This computation does not involve any nonlinear operation while considering nonlinear lens distortions.

\section{Experimental Results}

Two experimental results will be shown in this section. One is for synthesized images of experimental simulation, and the other is for actual images taken with a commerical CCD camera.

Fig. 8 shows a synthesized image assuming the positional relation between the object coordinates and the camera coordinates are given as the rotation angle with respect to $x, y$ and $z$ axes are $\pi / 8, \pi / 4$ and $\pi / 6$, respectively, and translation in $x, y$ and $z$ directions are $(20,30,500)$ in $\mathrm{mm}$. The focal length of the camera is $50 \mathrm{~mm}$. The calibration plane $G_{1}$ is given at $Z_{1}=0$, and $G_{2}$ at $Z_{2}=20 \mathrm{~mm}$. Two sets of 25 test points are given in each plane at $5 \times 5$ grid points.

From this synthesized image without image reading noise, by using the present method, the coordinates of the optical center $(-355.2,101.4,-338.9)$ in $\mathrm{mm}$ with respect to the object coordinate system and the focal length $50.0 \mathrm{~mm}$ which are exact values used in the synthesis of the image are obtained.

In this method, the main errors were introduced in readings of image points. Then, to the readings of image points we added Gaussian noises with a standard deviation of 1 pixel size. The average calibration errors in standard deviation for 100 samples were as follows: The error of the focal length was 0.26 $\mathrm{mm}$ for the true value of $50 \mathrm{~mm}$, those of the $x$ and $y$ components of the effective center were about 0.7 $\mathrm{mm}$ and that of $z$ component was $5.3 \mathrm{~mm}$ for a distance of about $500 \mathrm{~mm}$, and that of the optical center was about 5 pixels in both $u$ and $v$ directions.
Fig. 9 shows images of a block total sizes of which are $5.05 \mathrm{~cm} \times 5.05 \mathrm{~cm} \times 12.65 \mathrm{~cm}$. They are sequence of 20 images taken by a moving camera, and the first, 10 th and 20th images are shown. In each image 25 vertices are visible, which are labeled from 1 to 25 , and the bold lines show the sequence of those vertex points. The image coordinates of those points were read out manually.

The origin of the image coordinates $(u, v)=(0,0)$ was set to be random and off the screen to show that this technique does not depend on the set-up of the image coordinates.

The origin of the object coordinates $(X, Y, Z)=(0$, 0,0 ) was set at the vertex $25, X$-axis was on the edge including vertices 1 and 25 , and $Y$-axis on the edge including vertices 18 and 25 . This block can be interpreted to have four parallel planes to $X-Y$ plane, the first of which consists of the points $\{1,8,9,12,13,16$, $18,19,21,22,24,25\}$, the second $\{10,11,14,15,20,23\}$, the third $\{4,5\}$, and the fourth $\{2,3,6,7\}$.

In the experiments, we used the first plane as the first calibration plane, which includes 12 test points, and the fourth as the second calibration plane, which includes four test points. The physical pixel pitches on the CCD image sensor were given as $0.009836 \mathrm{~mm}$ in $u$ and $0.0092784 \mathrm{~mm}$ in $v$.

We solved the calibration problem with non-lensdistortion model given in Section 3 and 4 , and also with lens distortion model given in Section 5.

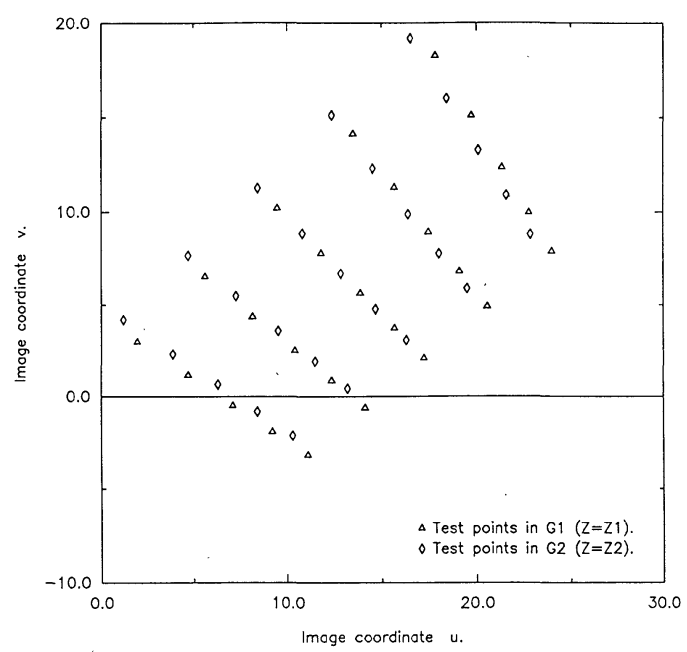

Fig. 8 Image of two calibration planes used in an experimental simulation 
Table 1 is a list of a part of calibration results for the image sequence. The images in the sequence which had not good quality to read out the vertex positions were skipped in the experiment and the result of which are not listed.

The effective center and the effective axis listed were obtained using non-distortion model. The results shows that the camera movement had constant rotational and translational velocities, which was the case of this experiment. To verify the positioning results, the calculated effective centers were fitted to the true movement trajectory of the camera, and

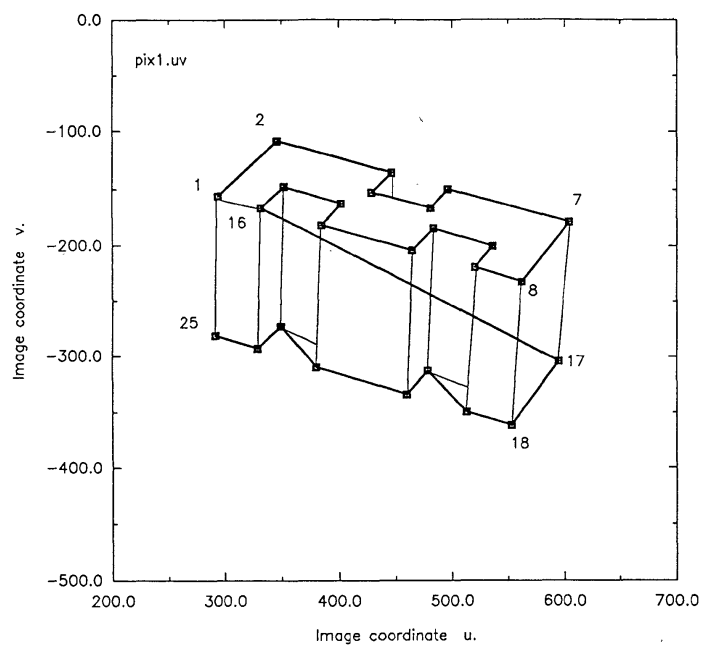

(a) First image in sequence

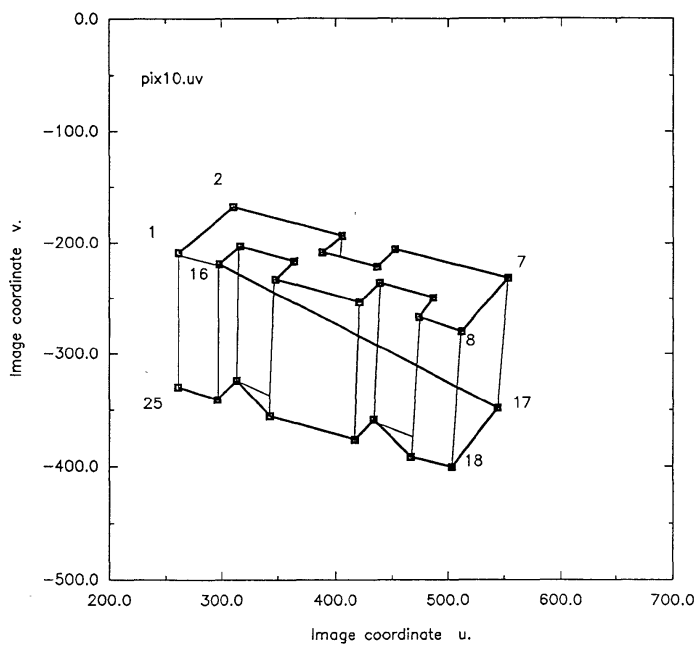

(b) 10th image in sequence evaluated the errors. Their standard deviations were about $3 \mathrm{~mm}$ in both $X$ and $Y$ directions and $10.1 \mathrm{~mm}$ in $Z$ direction with object distance of about $75 \mathrm{~cm}$. The values of effective center and axis obtained using the distortion model were slightly different from these values, but within $1 \%$ for all values. This is because the lens used in the experiments has high quality and small distortion, and also because these "effective" values are robust for small lens distortions.

Two sets of resulted values of focal lengths are listed, one was with non-distortion model and other was with distortion model. They must be constant through out the results within each respective set. The calibrated values with non-distortion model had a standard deviation of $0.29 \mathrm{~mm}$ for the average of $19.3 \mathrm{~mm}$, which was $1.5 \%$. That with distortion model has $0.11 \mathrm{~mm}$ of standard deviation for 19.2 $\mathrm{mm}$ of the average value $(0.6 \%)$. The value with distortion model was slightly improved, which also means the lens had good quality.

These calibrated values are fairly good in comparison with the results reported in the literatures.

\section{Conclusions}

In this paper, we examine the two plane calibration model in terms of perspective mapping, and present a new effective method by taking the advantage of the two plane method and modifying it based on the

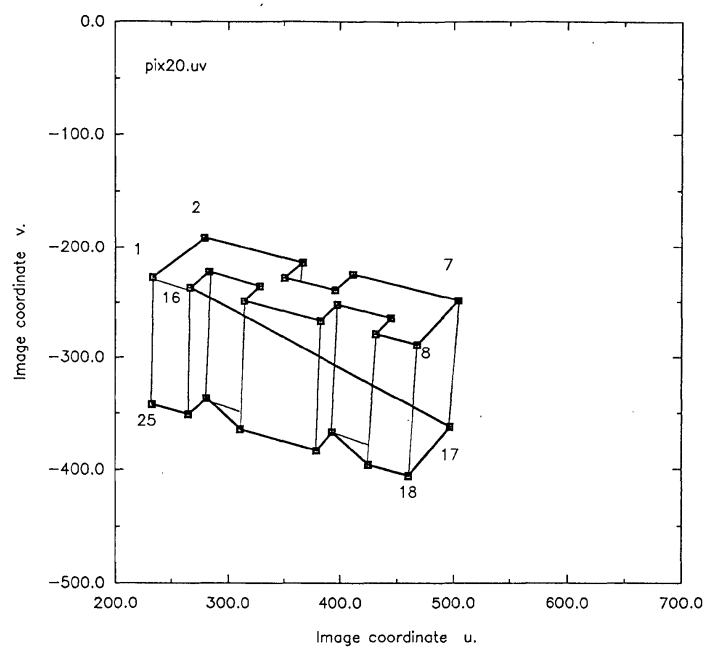

(c) 20th image in sequence

Fig. 9 Image sequence by an actual moving camera. (a) First image in the sequence, (b) 10th image, and (c) 20th image 
Table 1 Camera calibration results from images of Fig. 9. Focal lengths with (non-dist.) were by no-lens-distortion model, and those with (distortion) were by lens-distortion model

\begin{tabular}{|c|c|c|c|c|c|c|c|c|}
\hline \multirow{2}{*}{$\begin{array}{l}\text { Image } \\
\text { number }\end{array}$} & \multicolumn{3}{|c|}{ Effective center $(\mathrm{cm})$} & \multicolumn{3}{|c|}{ Effective axis } & \multirow{2}{*}{$\begin{array}{c}\text { Focal length } \\
\text { (non-dist.) } \\
\text { (mm) }\end{array}$} & \multirow{2}{*}{$\begin{array}{c}\text { Focal length } \\
\text { (distortion) } \\
\text { (mm) }\end{array}$} \\
\hline & $X$ & Y & Z & $g_{X}$ & $g_{Y}$ & $g_{7}$ & & \\
\hline 1 & 37.27 & 40.46 & -70.44 & -0.38 & -0.39 & 0.87 & 19.62 & 19.53 \\
\hline 2 & 37.24 & 40.01 & -70.97 & -0.39 & -0.39 & 0.87 & 19.70 & 19.57 \\
\hline 3 & 37.07 & 39.67 & -71.63 & -0.40 & -0.38 & 0.87 & 19.66 & 19.41 \\
\hline 4 & 38.41 & 39.15 & -71.93 & -0.41 & -0.38 & 0.85 & 19.81 & 19.53 \\
\hline 9 & 38.30 & 38.40 & -72.92 & -0.43 & -0.36 & 0.84 & 19.31 & 19.20 \\
\hline 10 & 35.46 & 38.06 & -73.25 & -0.44 & -0.35 & 0.84 & 19.10 & 19.01 \\
\hline 12 & 36.57 & 37.70 & -73.75 & -0.45 & -0.35 & 0.84 & 19.23 & 19.28 \\
\hline 13 & 39.41 & 37.18 & -74.54 & -0.46 & -0.35 & 0.84 & 18.90 & 19.10 \\
\hline 14 & 39.17 & 37.04 & -75.02 & -0.46 & -0.34 & 0.83 & 19.52 & 19.37 \\
\hline 15 & 34.97 & 36.85 & -75.84 & -0.46 & -0.33 & 0.83 & 19.13 & 19.20 \\
\hline 18 & 34.54 & 35.94 & -77.19 & -0.48 & -0.31 & 0.82 & 18.79 & 19.08 \\
\hline 20 & 34.14 & 35.46 & -77.83 & -0.48 & -0.31 & 0.81 & 19.30 & 19.20 \\
\hline
\end{tabular}

exact perspective imaging. It also takes the great advantage of the conventional two plane method that each of intrinsic and extrinsic camera parameters can be obtained by solving linear equations only, so that the solutions will be provided uniquely and stably.

As mentioned above, the main errors are introduced in readings of image points. The analysis of this kind of error and the stability conditions are the next problem. They must be verified under welldefined experimental protocols.

The performance tests under large lens distortions are also next problem.

\section{References}

1) H. A. Martins, J. R. Birk and R. B. Kelly: Camera Models Based on Data from Two Calibration Planes, Computer Graphics and Image Processing, 17, 173/180 (1981)

2) K. D. Gremban, C.E. Thorpe and T. Kanade: Geometric Camera Calibration using System of Linear Equations, Proc. IEEE International Conference on Robotics and Automation, no. 2, 562/567 (1988)

3) R. Y. Tsai: An efficient and Accurate Camera Calibration Technique for $3 \mathrm{D}$ Machine Vision, Proc. IEEE International Conference on Computer Vision and Pattern Recognition, 364/374 (1988)

4) A. Izaquire, P.Pu and J. Summers: A New Development in Camera Calibration Calibrating a Pair of Mobile Camera; Proc. IEEE International Conference on Robotics and Automation, 74/79 (1985)

5) G-Q Wei and S. D. Ma: Two Plane Camera Calibration: A Unified Model, Proc. IEEE International Conference on Computer Vision and Pattern Recognition, 133/138 (1991)

6) H.S. M. Coxeter: Projective Geometry, Second Edition, Springer Verlag (1987)

7) K. Deguchi : Projective Geometry for Computer Vision and Graphics [1] [4], Journal of SICE, 29-11 (1990) $\sim 30-3$ (1991) (in Japanese)
8) J. Weng, P. Cohen and M. Herniou: Calibration of Stereo Cameras using a Non-Linear Distortion Model Proc. 10th International Conference on Pattern Recog. nition, 246/253 (1990)

9) J. Weng, P. Cohen and M. Herniou: Camera Calibration with Distortion Models and Accuracy Evaluation, IEEE Transactions on Pattern Analysis and Machine Intelligence, PAMI-14-10, 965/980 (1992)

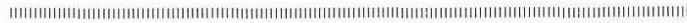
Koichiro DEGUCHI (Member)

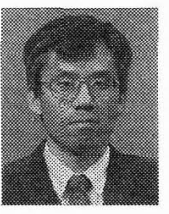

He received B. Eng., M. Eng. and Dr. Eng. degrees from University of Tokyo in 1974, 1976 and 1980, respectively. From 1984 to 1987 , he was an associate professor of Department of Information Engineering, Yamagata University, and sice 1988, he is an associate professor of Department of Mathematical Engineering and Information Physics, Unversity of Tokyo. His recent research interests include computer vision, signal processing and multiprocessor architecture for them.

\section{Iwao Morishita (Member)}

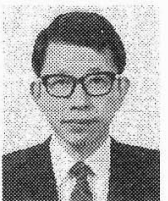

He received the B. Eng., M. Eng. and Dr. Eng. degrees from University of Tokyo in 1957, 1964 and 1968, respectively. In 1973 he stayed at the Artificial Intelligence Center of SRI International as a visiting researcher. Since 1980 he has been a professor at the Department of Mathematical Engineering and Information Physics, Faculty of Engineering, University of Tokyo. His reseach interests include image processing, multiprocessor architecture and system software for parallel processing. 